\title{
SOURCE TERM MODEL FOR STEADY MICRO JETS IN A NAVIER-STOKES COMPUTER CODE
}

\author{
Kenrick A. Waithe† \\ NASA Langley Research Center \\ Hampton, VA
}

\begin{abstract}
A source term model for steady micro jets was implemented into a non-proprietary Navier-Stokes computer code, OVERFLOW. The source term models the mass flow and momentum created by a steady blowing micro jet. The model is obtained by adding the momentum and mass flow created by the jet to the Navier-Stokes equations.

The model was tested by comparing with data from numerical simulations of a single, steady micro jet on a flat plate in two and three dimensions.

The source term model predicted the velocity distribution well compared to the two-dimensional plate using a steady mass flow boundary condition, which was used to simulate a steady micro jet. The model was also compared to two three-dimensional flat plate cases using a steady mass flow boundary condition to simulate a steady micro jet. The threedimensional comparison included a case with a grid generated to capture the circular shape of the jet and a case without a grid generated for the micro jet. The case without the jet grid mimics the application of the source term. The source term model compared well with both of the three-dimensional cases. Comparisons of velocity distribution were made before and after the jet and Mach and vorticity contours were examined.

The source term model allows a researcher to quickly investigate different locations of individual or several steady micro jets. The researcher is able to conduct a preliminary investigation with minimal grid generation and computational time.
\end{abstract}

\footnotetext{
$\div$ Aerospace Engineer. Configuration Aerodynamics Branch. Research \& Technology Directorate. Member AIAA.

This material is declared a work of the U.S. Government and is not subject to copyright protection in the United States
}

\section{Introduction}

Boundary layer ingesting (BLI) serpentine (Sduct) inlets have the potential benefits of reduced drag, size, and weight by eliminating the boundary layer diverter and shortening the inlet duct. Two drawbacks of BLI S-duct inlets are increased turbofan engine-face distortion and reduced pressure recovery. Low-profile vortex generating (VG) vanes have shown significant improvement in turbofan engine-face distortion in the design of compact aircraft inlets [1]. Vortex generating vanes can increase the radar signature of an inlet and can also be difficult to maintain. The use of non-intrusive vortex generating techniques such as micro jets, are being investigated at NASA Langley Research Center by Gorton et al. [2] and other institutions.

Computational fluid dynamics (CFD) is a tool that can be used in conjunction with experiments to design new inlets. Before CFD can be used, researchers have to be sure that $C F D$ analysis can model the complex flows associated with the vortices created by a vortex generating device such as vanes and micro jets. Allan et al. [3] have shown a comparison between a CFD analysis and experimental data of a low-profile vortex generator vane on a flat plate. Their results indicate that CFD analysis can accurately predict various aspects of the resulting vortex including size, shape, location, and decay. While the work conducted by Allan et al. was significant, the method used to model the vane has many drawbacks. In particular, the small size of the vane required very fine grids to model the vane and the region immediately surrounding the vane. The result was an increase in the number of grids required to model the entire flow and with an increase in grids comes an increase in computational time. Modeling individual micro jets also results in an increase in grid size and computational time. As the number of vortex generating devices is increased from one to possibly twenty, a more efficient method must be used.

A source term model for an array of vortex generating vanes was developed by Bender et al. [4] and implemented by Waithe [5] into the ReynoldsAveraged Navier-Stokes (RANS) CFD code OVERFLOW version $1.8 \mathrm{~s}$ [6], which is a nonproprietary CFD code. The vane source term model was the first of three models to be implemented into OVERFLOW. Once complete the user will have a 
choice of using a model for vanes, steady micro jets, and pulsing micro jets. These models will allow the user to quickly investigate vortex generating devices.

This paper describes the implementation of the source term model for steady blowing micro jets in the Reynolds Averaged Navier-Stokes (RANS) CFD computer code OVERFLOW version 1.8s. In addition, this report presents the results of CFD analysis utilizing the source term model on a twodimensional (2D) flat plate modeling a single steady micro jet. The results are compared to a CFD analysis where a constant mass flow boundary condition is used at the surface to model the micro jet. Also, CFD analysis using the source term model to model a steady micro jet on a three-dimensional (3D) flat plate is compared to two computational cases of the same flat plate with a steady mass flow boundary condition to model a steady micro jet. The first case utilizes a grid generated to capture the shape of the micro jet. The second case does not utilize a grid to capture the shape of the micro jet, but applies the mass flow boundary condition over a rectangular region of the existing grid. This mimics the application of the source term model.

\section{$\underline{\text { Nomenclature }}$}

A cross-sectional area

$\mathrm{A}_{\mathrm{i}} \quad$ cell area

$\mathrm{A}_{\text {jet }} \quad$ jet area

$\mathrm{A}_{\text {tot }} \quad$ total cell area of source term application

BC48 steady mass flow boundary condition

c calibration constant

dA differential cross-sectional area

$\mathrm{K}$ cross-stream kinetic energy

M Mach number

MFR $_{\text {jet }}$ jet mass flow rate

$\mathrm{S}_{1} \quad$ mass flow term

$\mathrm{S}_{\mathrm{i}} \quad$ momomentum term

$\mathrm{S}_{5} \quad$ energy term

$\hat{t} \quad$ unit vector normal to jet

$\mathrm{t}_{\mathrm{i}} \quad$ component $\mathrm{i}$ of vector $\hat{t}$

u component 1 of $\bar{U}$

$\mathrm{U}_{\partial} \quad$ free stream velocity

$\bar{U} \quad$ local velocity vector

v component 2 of $\bar{U}$

w component 3 of $\bar{U}$

$\mathrm{w}_{\mathrm{jet}} \quad$ jet velocity

$\mathrm{x} \quad$ axial direction

y cross stream direction

$\mathrm{z}$ normal direction

$\partial \quad$ angle of attack of jet to local flow

$\partial \quad$ angle of incidence of jet to surface

$\partial$ density $\partial_{\partial} \quad$ free stream density

$\partial \quad$ stream-wise vorticity

$\partial v$ differential of $v$

$\partial w \quad$ differential of $w$

$\partial y \quad$ differential of $y$

$\partial \mathrm{z}$ differential of $\mathrm{z}$

\section{Abbreviations}

2D two-dimensional

3D three-dimensional

BLI boundary layer ingestion

CFD computational fluid dynamics

RANS Reynolds Averaged Navair-Stokes

SA Spalart and Allmaras

VG vortex generator

\section{Numerical Method}

The basic methodology of the model is to introduce mass flow, momentum and energy terms to the mass flow, momentum, and energy equations representative of the mass flow, momentum, and energy that are added by a steady blowing micro jet. The terms are given in equations 1,2 and 3 .

$$
\begin{aligned}
& S_{1}=c \frac{A_{i}}{A_{t o t}} \infty_{\infty} A_{j e t} M F R_{j e t} U_{\infty} \\
& S_{i}=S_{1} U_{x} t_{i} \quad(i=2,3,4) \\
& S_{5}=\underset{i=2}{4} S_{i} t_{i} U_{\infty}
\end{aligned}
$$

In equations 1 to $3, S_{1}$ is the mass flow term, $S_{i}$ is the momentum term, $S_{5}$ is the energy term, $c$ is a calibration constant, $A_{i}$ is the cell area, $A_{\text {tot }}$ is the total cell area, $A_{\text {jet }}$ is the area of the jet, $\partial_{\partial}$ is the free stream density, $U_{\partial}$ is the free stream velocity, MFR $_{\text {jet }}$ is the mass flow rate of the jet, and $t_{i}$ is the directional component of the unit vector $\hat{t}$.

The source term model was implemented into the CFD code OVERFLOW version 1.8s. OVERFLOW solves the steady, compressible, Reynolds-Averaged Navier-Stokes (RANS) equations using the diagonal scheme of Pulliam and Chaussee [7]. The RANS equations are solved on structured grids using the overset grid framework of Steger et al. [8], which allows for complex geometries. The source term model is invoked in OVERFLOW by specifying a region of cells containing the steady micro jet to be modeled as shown in figure 1 . In particular, the cells that enclose the area of the jet and the second row of cells from the surface are selected to add the 
momentum and mass flow of the jet. The first row is not selected because the source term model is not a boundary condition and the first row of cells in OVERFLOW is reserved for boundary conditions. Since the cells are typically very dense in the boundary layer, the application on the second row of cells is sufficient.

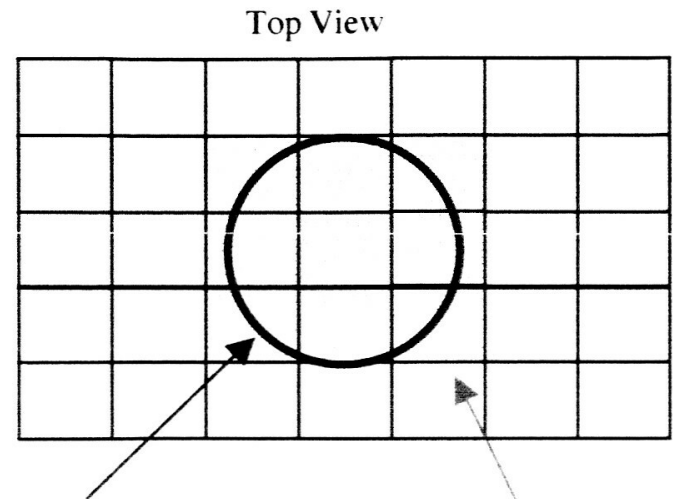

jet being modeled

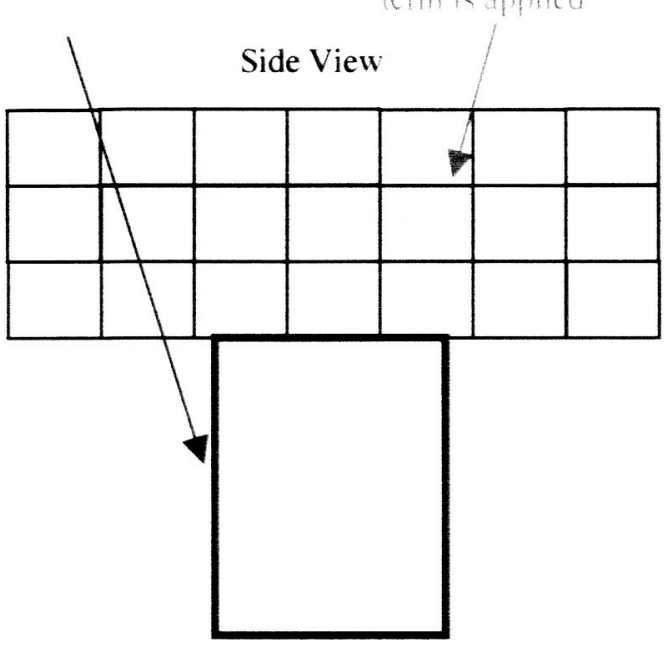

Figure 1. Schematic of cells selected for application of source term model.

The user also specifies the calibration constant $c$, jet mass flow rate, MFR $_{\text {jet }}$ based on the free stream flow conditions, the jet area, $A_{\text {jet }}$, the angle of attack between the local flow and the micro jet, $\partial$, and the angle of incidence to the surface, $\partial$. Figure 2 shows a schematic with the orientation of the jet to a surface.

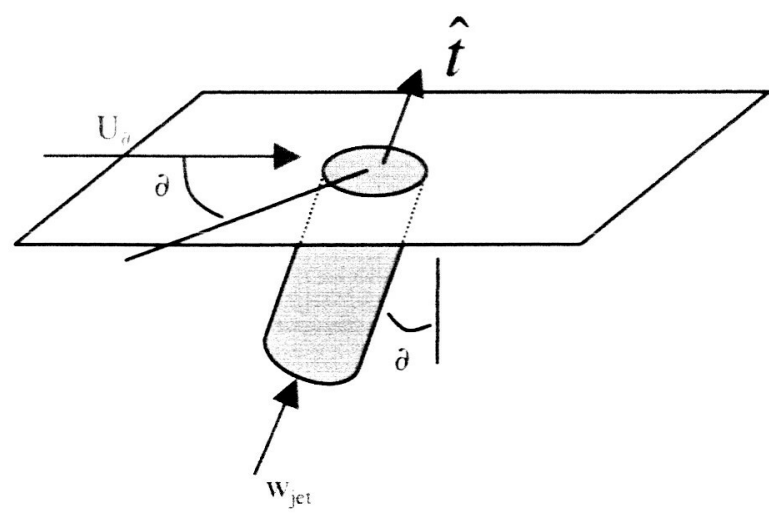

Figure 2. Schematic of jet orientation.

\section{D Flat Plate Study}

The source term model was tested by running simulations on a 2D flat plate using the source term to model a steady micro jet. The model was compared to simulations on a $2 \mathrm{D}$ flat plate using a steady mass flow boundary condition to model a steady micro jet. The grid for the flat plate used in this study is shown in figure 3 and comprises a single block grid with a total of 9,333 grid cells.

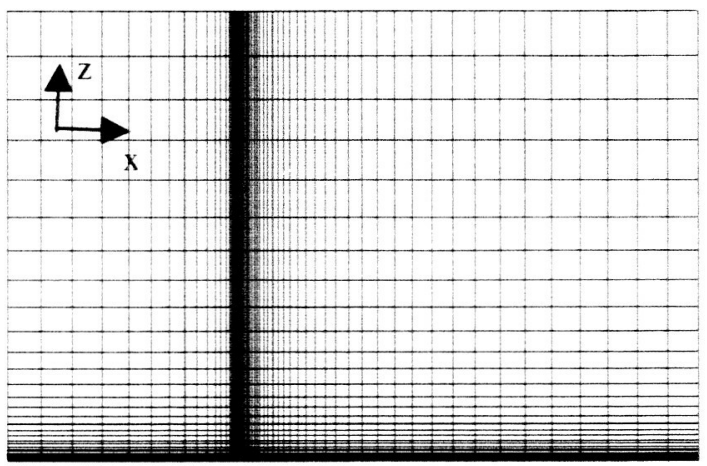

Figure 3. Computational domain for $2 D$ flat plate study.

The grid is dimensionless. The jet modeled has an area, $\mathbf{A}_{\mathrm{jel}}=0.015$. The source term model was applied with an angle of attack, $\dot{\partial}=0^{\circ}$ and an angle of incidence, $\partial=0^{\circ}$. If the angle of incidence or angle of attack were not zero, a grid for the jet would have been generated for use with the steady mass flow boundary condition in order to generate the desired angles of attack and incidence. The steady mass flow boundary condition does not allow the user to specify an angle of attack or incidence; they are both zero by default. The source term model allows the user to investigate varying angles of attack and incidence 
with out having to generate a grid for the jet being modeled. All simulations were run at a free stream Mach number, $\mathrm{M}_{\partial}=0.2$, a Reynolds number, $\mathrm{Re}=1 \times 10^{6}$, and a jet mass flow rate, $\mathrm{MFR}_{\mathrm{jet}}=1.0$.

The multi-grid acceleration option of OVERFLOW was used to improve the convergence of the steady-state solution. All simulations were run using the one-equation Spalart and Allmaras (SA) model [9]. The SA model was used because of its simplicity.

OVERFLOW was run on a Compaq Alpha 500 $\mathrm{MHz}$ machine for this study. A total of nine runs were completed with the source term model to investigate the effects of the calibration constant and one run with the steady mass flow boundary condition. The total run time for each case was approximately one minute.

\section{D Flat Plate Results}

The results of the source term model simulations were compared to simulations using a steady mass flow boundary condition. The source term model was calibrated before comparisons were made. The model was calibrated by plotting the effects of the normalized, cross-stream kinetic energy, $\mathrm{K}$, downstream of the jet as the calibration constant is increased. As the calibration constant is increased, the cross-stream kinetic energy downstream of the source term should asymptote to a constant value. The source term model is correctly calibrated by using values of the calibration constant that occur in the asymptotic region of the cross-stream kinetic energy plot. The normalized, cross-stream kinetic energy is given by equation 4 .

$$
K=\frac{\int_{A} \int\left(v^{2}+w^{2}\right) d A}{\iint_{A} u^{2} d A}
$$

In equation $4, u, v$, and $w$ are the components of the local velocity vector, $\bar{U}, \partial$ is the density of the flow field, and $A$ is the area of the cross-section over which the cross-stream kinetic energy is integrated. Figure 4 shows the square root of the normalized, cross-stream kinetic energy, $K^{1 / 2}$, versus the calibration constant, c. For comparisons with the steady mass flow boundary condition, a calibration constant of $c=3.8$ was used,

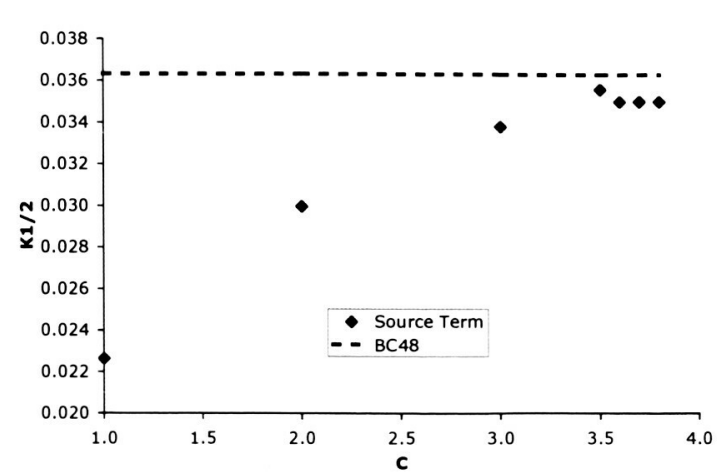

Figure 4. Square root of cross-stream kinetic energy vs. calibration constant, $c$.

Figure 5 and 6 shows the mach contours of the source term and steady mass flow boundary condition, respectively.

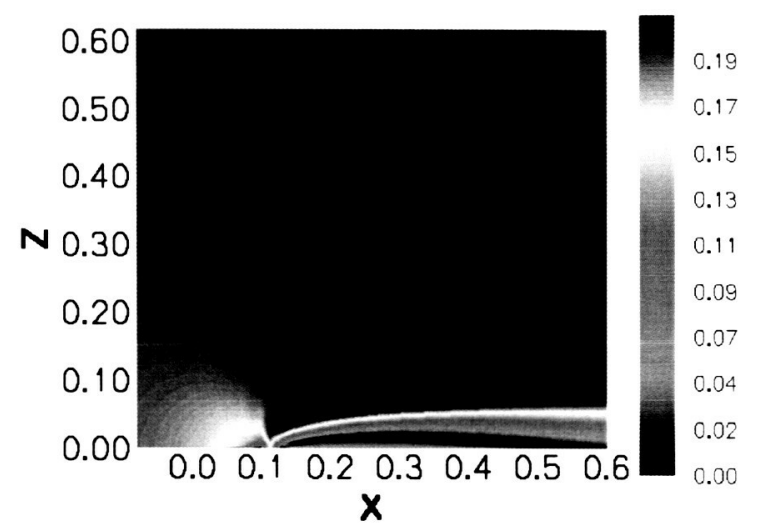

Figure 5. Mach contours using the source term model.

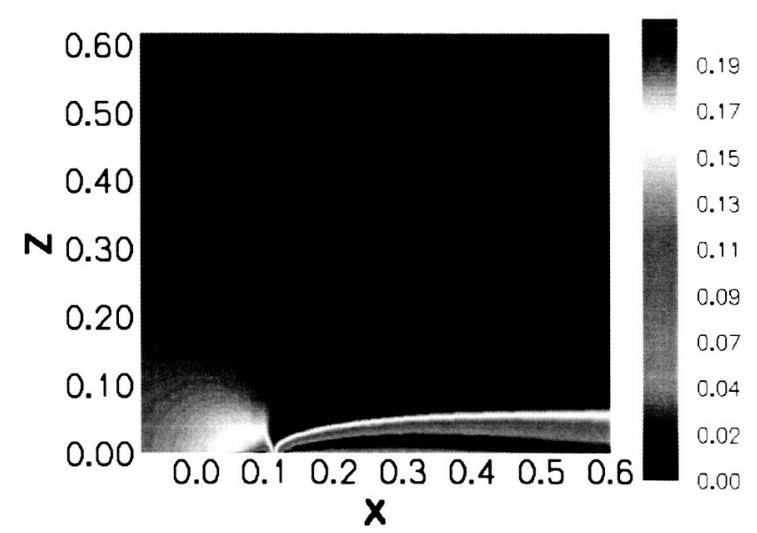

Figure 6. Mach contours using the steady mass flow boundary condition.

Figures 7 and 8 show close ups of the jet with Mach contours and velocity vectors of the source term model and steady mass flow boundary condition, 
respectively. The source term model does a very good job in reproducing the effects of the steady mass flow boundary condition.

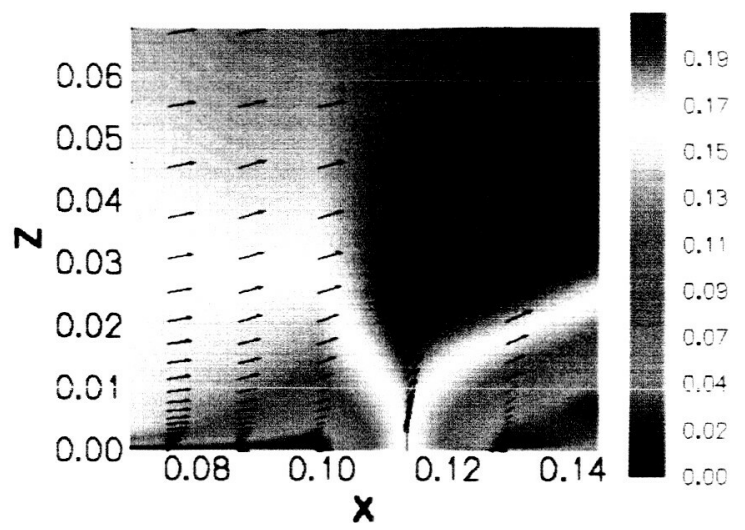

Figure 7. Close up of jet with Mach contour using the source term model.

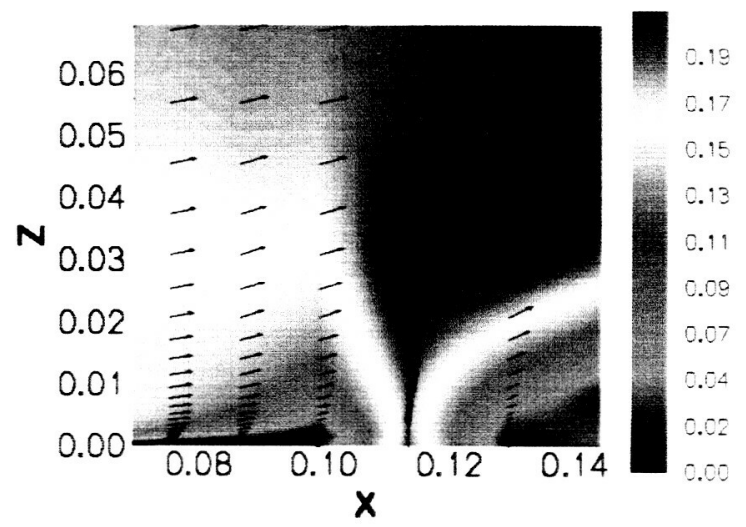

Figure 8. Close up of jet with Mach contours using the steady mass flow boundary condition.

The velocity profile at four different locations was examined to evaluate the effect the source term model had on the flow field compared to the steady mass flow boundary condition. Figures 10-13 show the velocity profiles caused by the source term model and the steady mass flow boundary condition. In figures 9-12, the steady mass flow boundary condition is labeled as BC48, which is its OVERFLOW name. At $x=0.05$ the velocity profiles of the source term and steady mass flow boundary condition are identical, which indicates the source term model and steady mass flow boundary condition produce similar effects upstream of the jet. The velocity profiles are only slightly different at locations immediately after the jet and very close to the surface. As the flow field moves away from the surface and away from the jet, the profiles are identical. The discrepancy between the profiles close to the surface could be attributed to the different locations where the source term and steady mass flow boundary condition are applied. In particular, the steady mass flow boundary condition is applied at the surface, while the source term is applied one cell away from the surface.

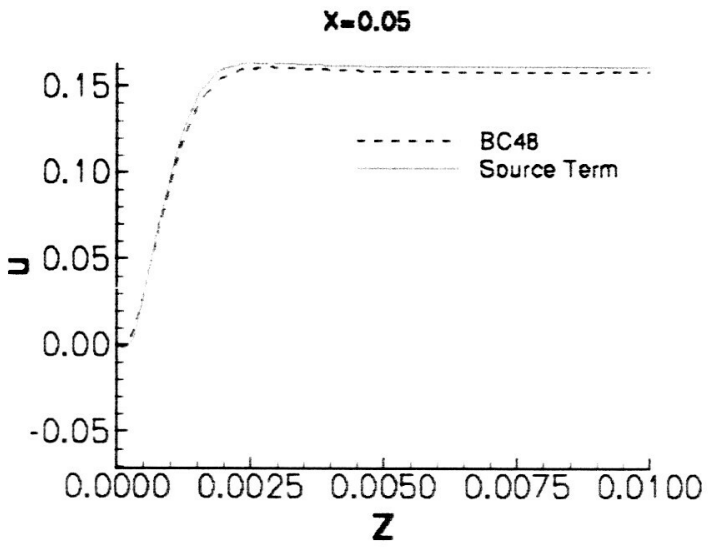

Figure 9. Velocity distribution at $x=0.05$.

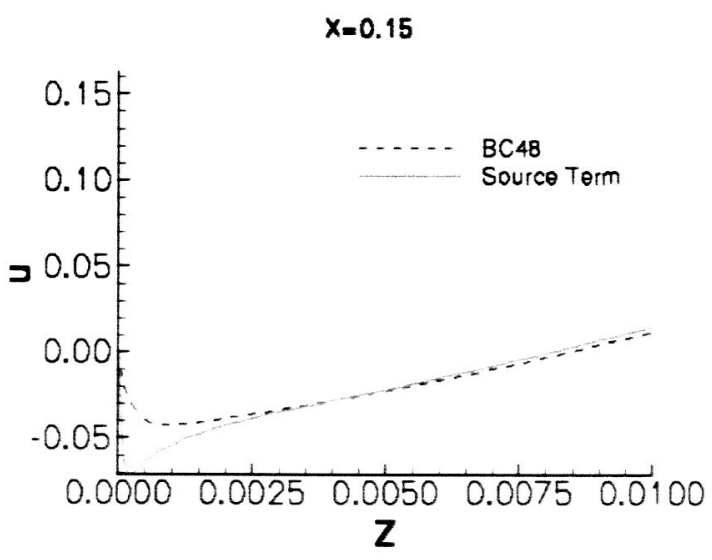

Figure 10. Velocity distribution at $x=0.15$.

$x=0.17$

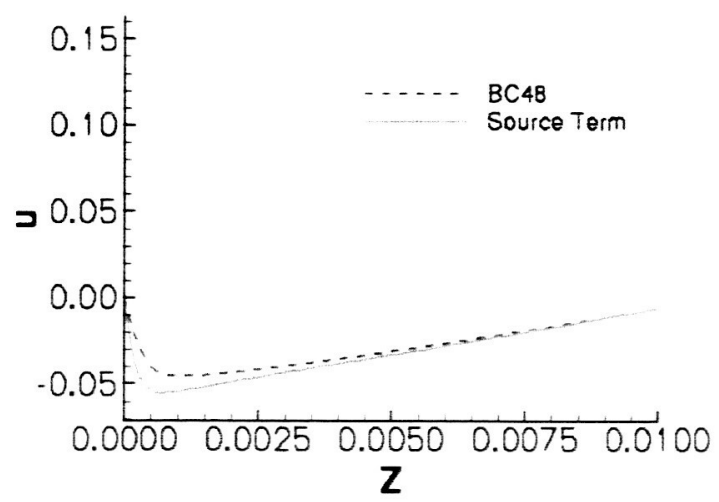

Figure 11. Velocity distribution at $x=0.17$. 
$x=0.19$

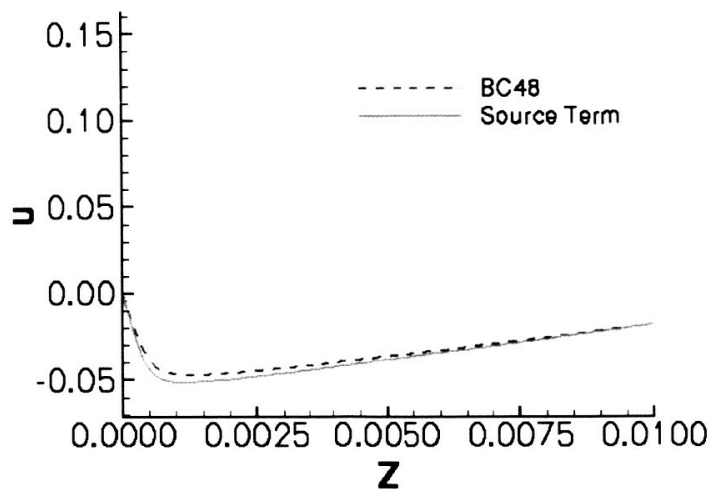

Figure 12. Velocity distribution at $x=0.19$.

A representative flow residual history of both the source term model and steady mass flow boundary condition is shown in figure 13 .

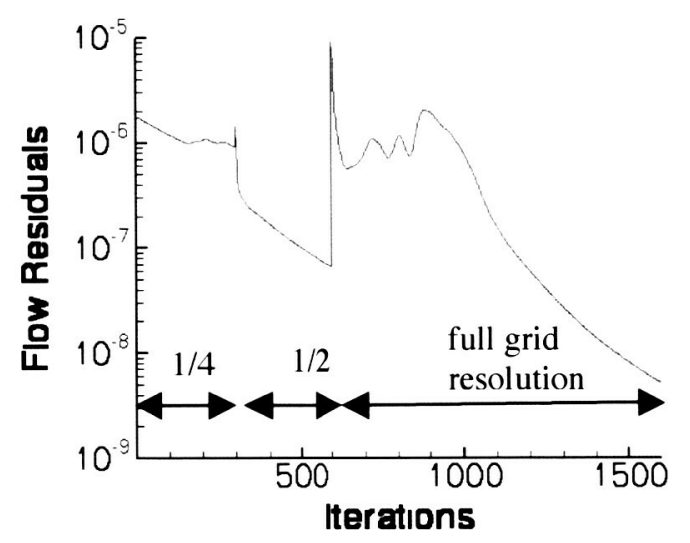

Figure 13. Flow residuals history.

The residual history is used to track the convergence of the CFD solution. A residual drop of several orders of magnitude is expected from the $1 / 4$ grid resolved solution to the full grid resolved solution of a converged solution. The solution has dropped close to three orders of magnitude.

\section{$\underline{\text { 3D Flat Plate Study }}$}

The source term model was also tested by running simulations on a $3 \mathrm{D}$ flat plate using the source term to model a steady micro jet. The model was compared to two simulations on a $3 \mathrm{D}$ flat plate using a steady mass flow boundary condition to model a steady micro jet. One of the simulations using the steady mass flow boundary condition used additional grids to capture the shape of the micro jet. The other simulation using the steady mass flow boundary condition did not use additional grids to capture the shape of the micro jet.

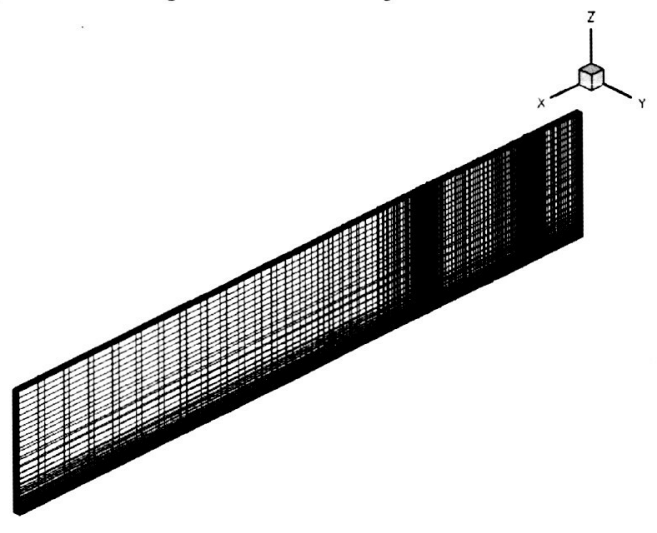

Figure 14. Computational domain for $3 D$ flat plate study.

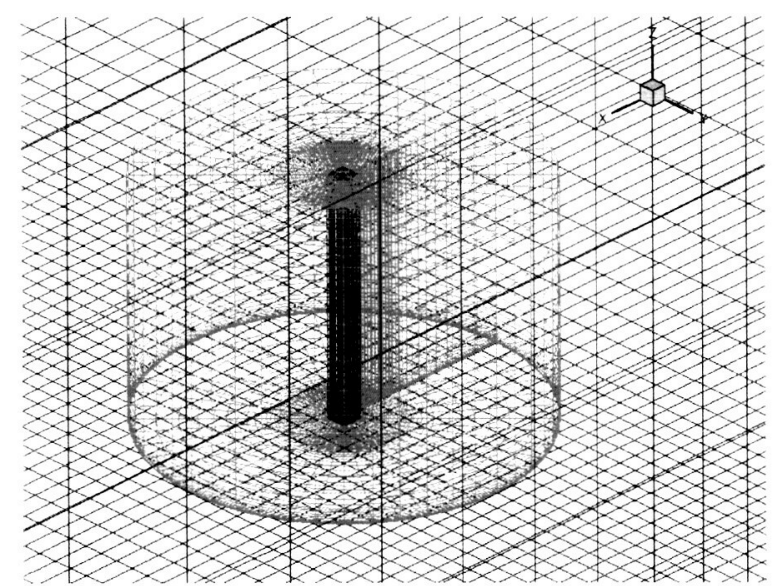

Figure 15. Close up of computational domain for steady mass flow boundary condition with jet grid.

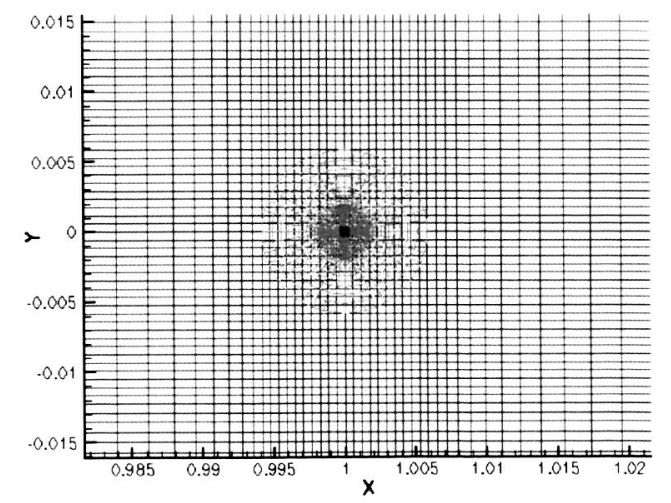

Figure 16. Top view, close up of computational domain for steady mass flow boundary condition with jet grid.

The boundary condition was applied to rectangular grid cells similar to the application of the source term 
model. The grids used in this study are shown in figures 14-16. The grid used for the source term model and the steady mass flow boundary condition with out the jet grid comprises a single block grid with a total of 698,139 grid cells. The grid used for the steady mass flow boundary condition with the jet grid comprises the single block grid used by the source term model $(698,139$ grid cells), a cylindrical grid for capturing the shape of the jet $(48,461$ grid cells) and an axis grid (2,783 grid cells) for a total of 749,383 grid cells. The axis grid is necessary to remove the singularity created by the cylindrical grid.

The grids are dimensionless. The jet modeled has an area, $A_{\text {jet }}=0.000106$. The source term model was applied with an angle of attack, $\partial=0^{\circ}$ and an angle of incidence, $\partial=0^{\circ}$. All simulations were run at a free stream Mach number, $\mathbf{M}_{i}=0.2$, a Reynolds number, $R e=1 \times 10^{6}$, and a jet mass flow rate, $\mathrm{MFR}_{\mathrm{jet}}=1.0$.

The multi-grid acceleration option of OVERFLOW was used to improve the convergence of the steady-state solution. All simulations were run using the one-equation Spalart and Allmaras (SA) model [9]. The SA model was used because of its simplicity.

OVERFLOW was run on a Compaq Alpha 500 $\mathrm{MHz}$ machine for this study. A total of three runs were completed with the source term model and the steady mass flow boundary condition. The total run time for each case was approximately ten minutes.

\section{$\underline{\text { 3D Flat Plate Results }}$}

The results of the source term model simulations were compared to two simulations using a steady mass flow boundary condition to model a steady micro jet. One of the simulations using the steady mass flow boundary condition utilized a grid for capturing the shape of the jet, while the other did not.

Figures 17-19 show the Mach contours of all three cases at the centerline of the jet, $y=0$. The source term model has features of both of the simulations with the steady mass flow boundary condition. In particular, the jet looks more like the simulation with the grid generated for the jet, but the region immediately following the jet looks more like the simulation without the grid generated for the jet.

Figures 20-23 show the centerline $u$ velocity distribution for all cases. The steady mass flow boundary condition is noted as BC48 on the plots. The velocity distributions are identical at $x=0.985$ as expected, which indicates very the three methods produce similar effects upstream of the jet.
Immediately after the jet, at $x=1.015$, the source term model has a profile that is similar to the steady mass flow boundary condition with the jet grid close to the surface $(\mathrm{z}=0)$.

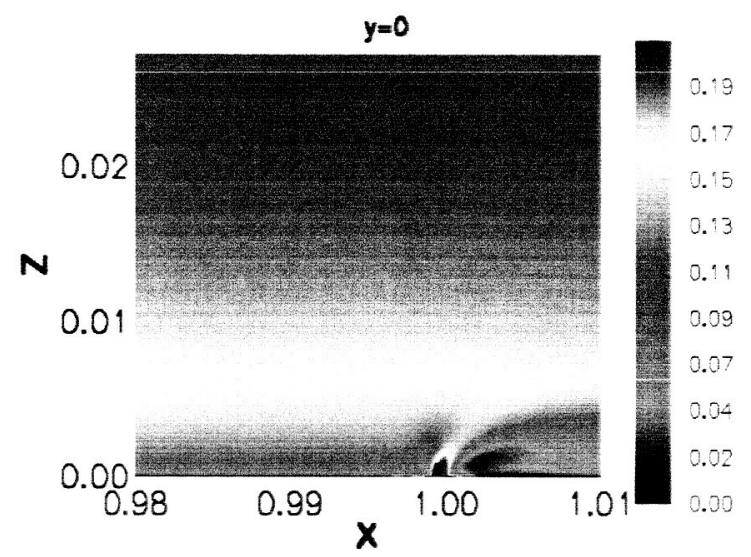

Figure 17. Mach contours using the source term model.

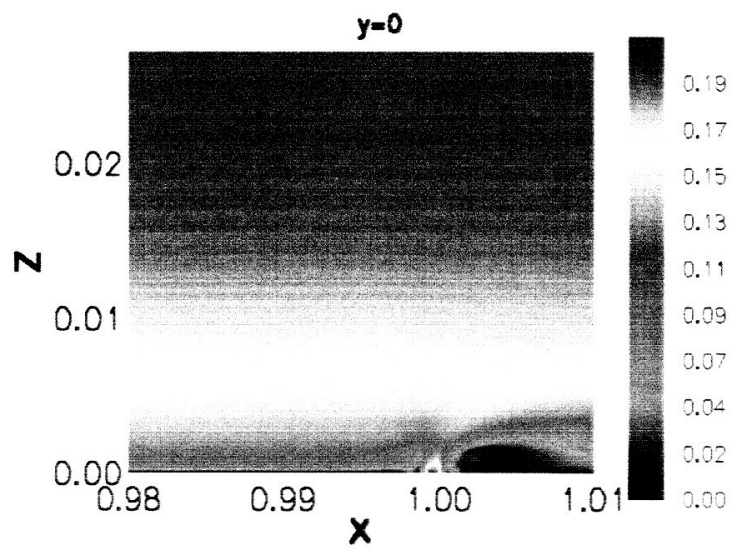

Figure 18. Mach contours using the steady mass flow boundary condition without jet grid.

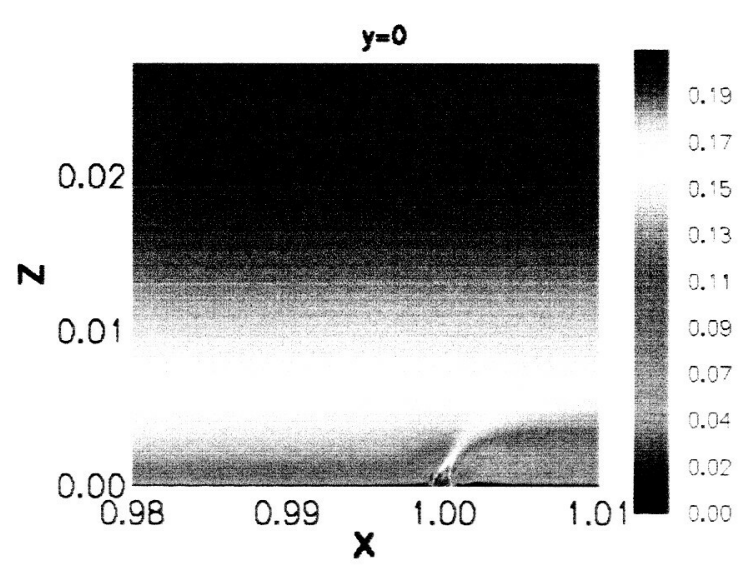

Figure 19. Mach contours using the steady mass flow boundary condition with the jet grid. 
However, at $\mathrm{x}=1.015$, the source term model has a profile similar to the steady mass flow boundary condition without the jet grid farther away from the surface. As the flow field is examined further down field, at $x=1.045$, the source term model is almost identical to the steady mass flow boundary condition with out the jet grid.

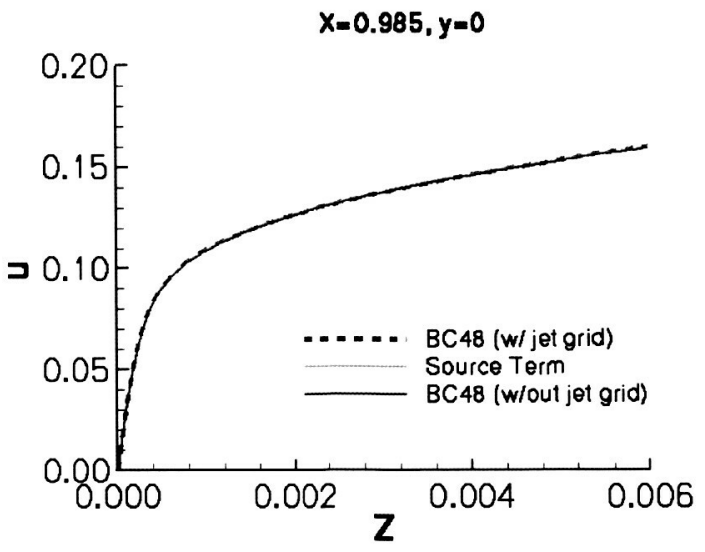

Figure 20. $u$ velocity distribution at $x=0.985, y=0$.

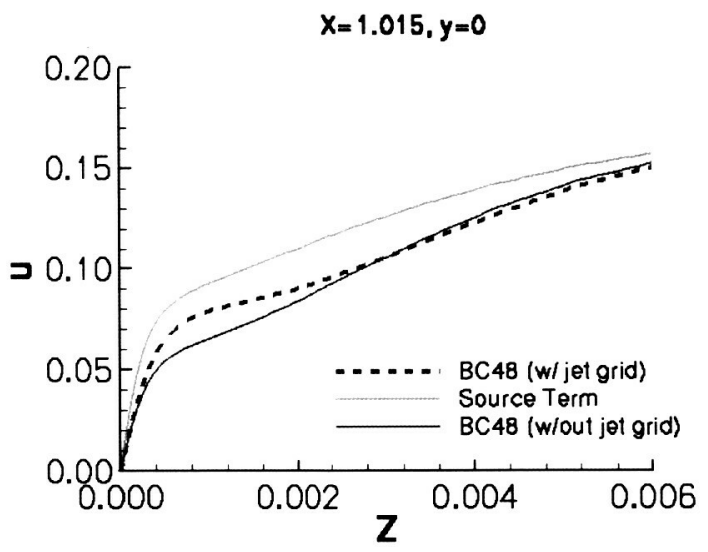

Figure 21. $u$ velocity distribution at $x=1.015, y=0$.

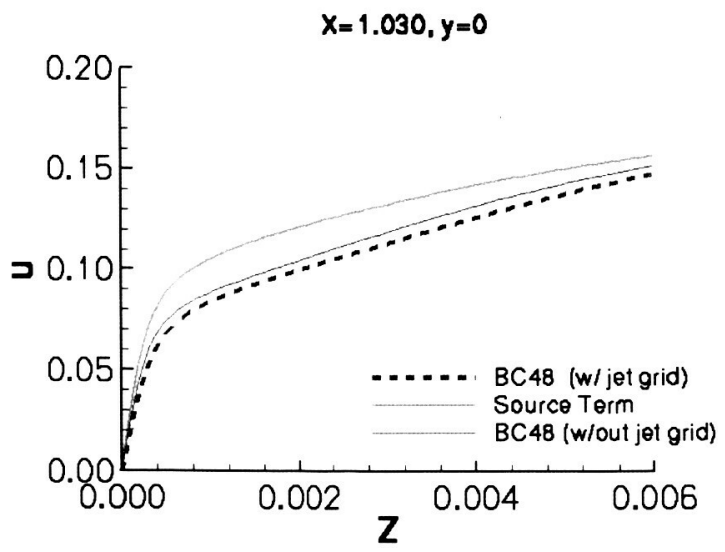

Figure 22. $u$ velocity distribution at $x=1.030, y=0$.

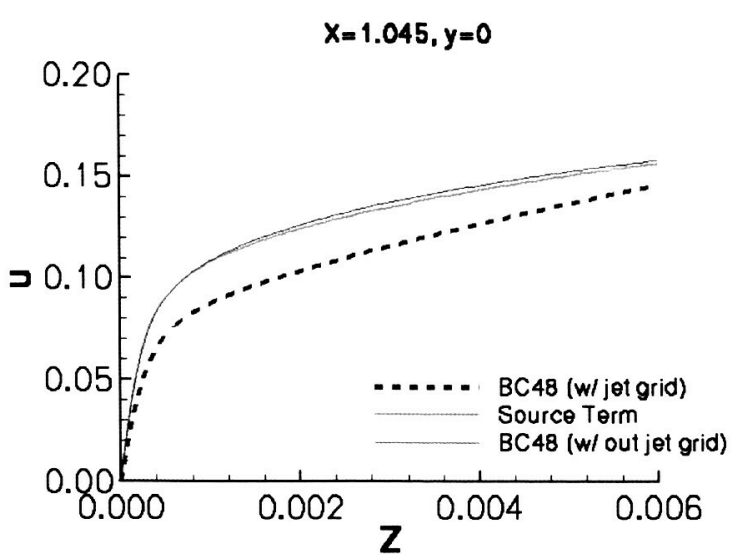

Figure 23. $u$ velocity distribution at $x=1.045, y=0$.

Figures 24-26 show the $u$ velocity contours at $\mathrm{x}=1.015$ for the source term model and steady mass flow boundary condition cases.

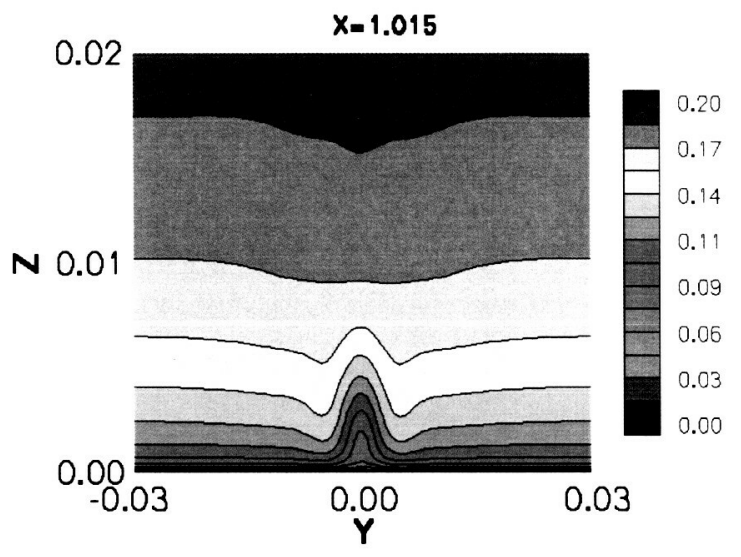

Figure 24. $u$ velocity contours of the source term model.

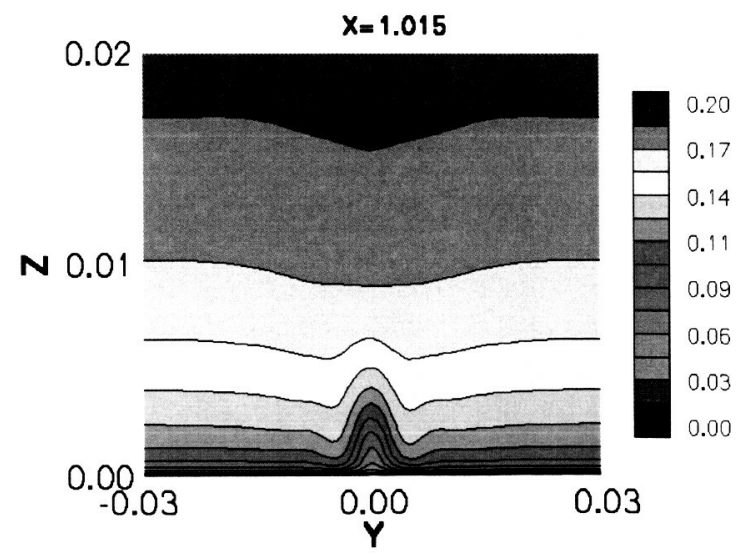

Figure 25. u velocity contours of the steady mass flow boundary condition without jet grid. 


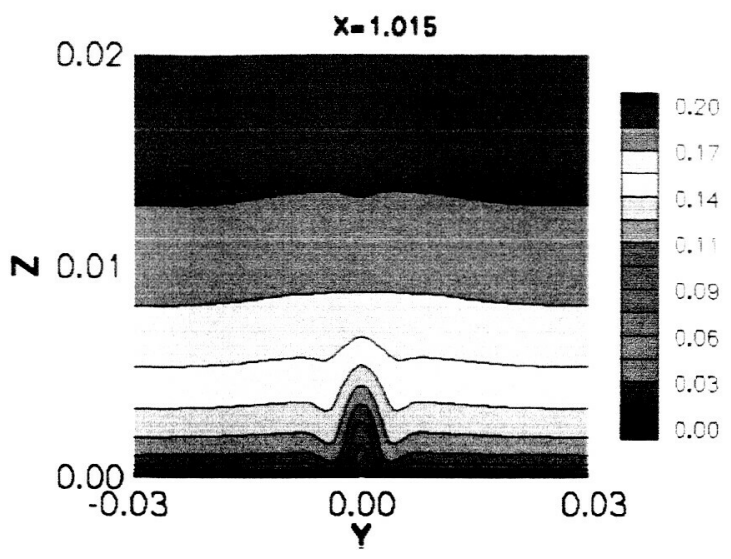

Figure 26. $u$ velocity contours of the steady mass flow boundary condition with jet grid.

The source term model is similar to the steady mass flow boundary condition with the jet grid close to the wall and to the steady mass flow boundary condition without the jet grid far from the wall.

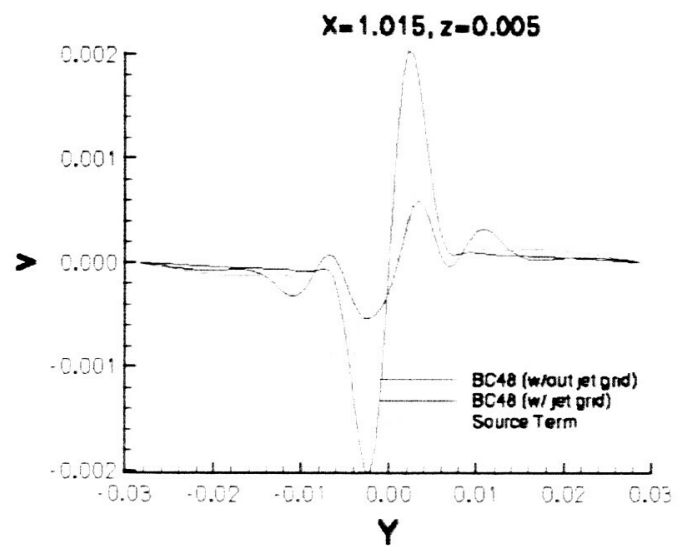

Figure 27. $v$ velocity distribution at $x=1.015$. $z=0.005$.

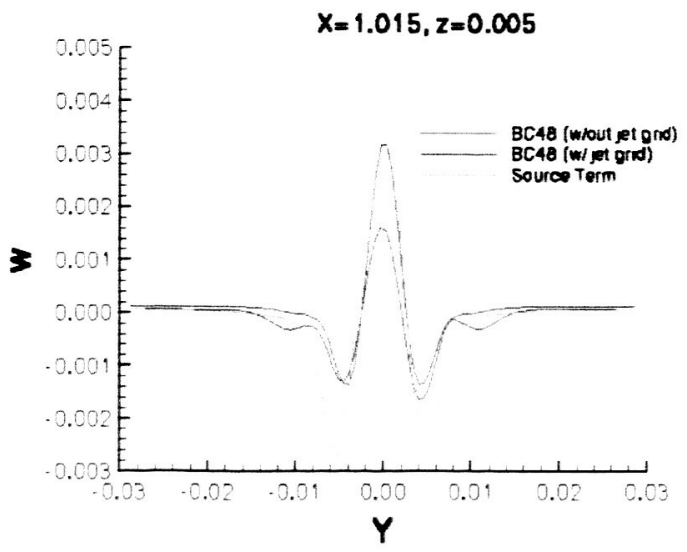

Figure 28. $w$ velocity distribution at $x=1.015$. $z=0.005$.
Figures 27 and 28 show the $v$ and $w$ velocity distribution at $\mathrm{x}=1.015$ and $\mathrm{z}=0.005$, respectively. The source term model does a fairly good job in modeling the shape of the $v$ and $w$ velocity distribution curves at the location specified.

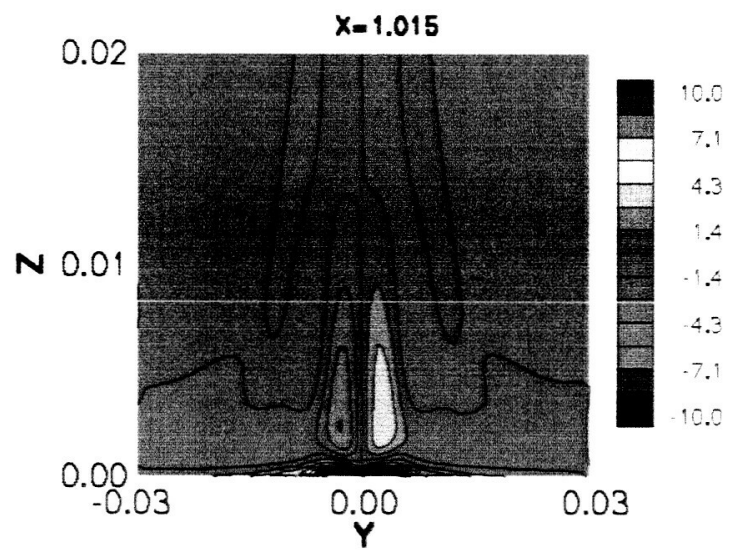

Figure 29. Cross-stream vorticity, $\partial$, for source term model.

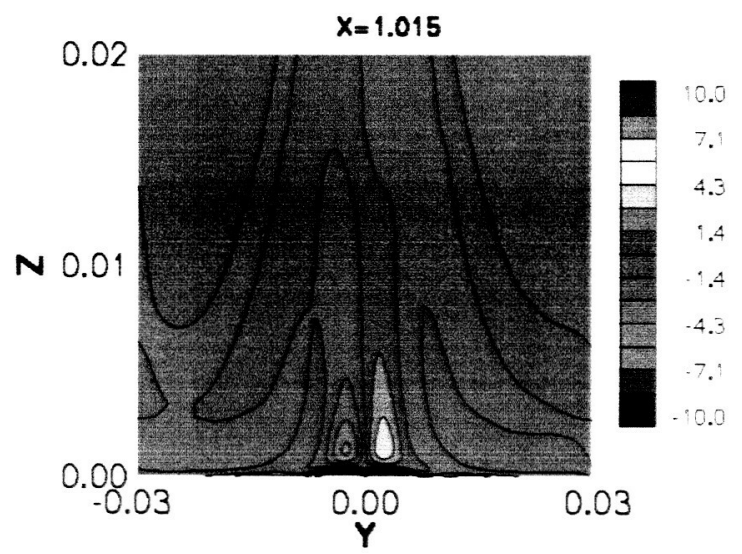

Figure 30. Cross-stream vorticity, $\partial$. for steady mass flow boundary condition without jet grid.

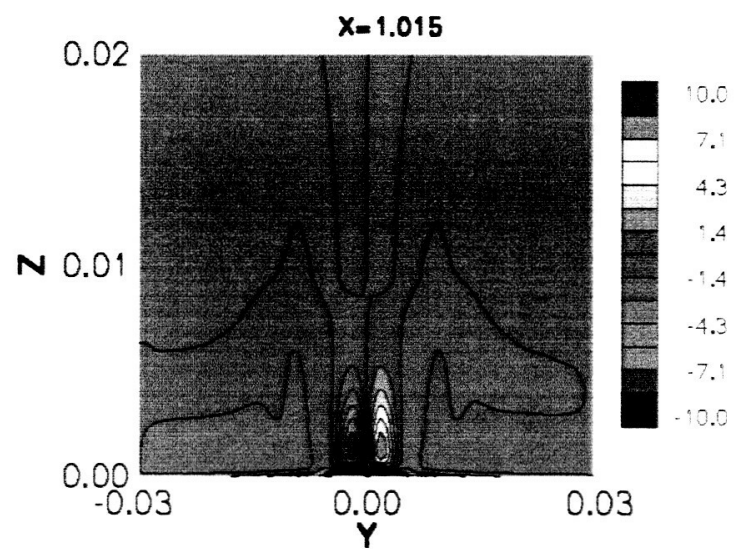

Figure 31. Cross-stream vorticity, $\dot{\partial}$, for steady mass flow boundary condition with jet grid. 
The cross-stream vorticity, $\partial$, was calculated for all three cases. The vorticity is calculated using equation 5 .

$$
-=\frac{-v}{-z}-\frac{-w}{-y}
$$

Figures 29-31 show the cross-stream vorticity for all three cases at $x=1.015$ (immediately after the jet). The source term model captures the vortical flow region fairly well, but the intensity is not as strong as the steady mass flow flow boundary condition with the jet grid.

\section{Conclusions}

In general, the source term model compared well with the steady mass flow boundary condition on both the 2D and 3D flat plates and is a viable alternative to gridding the jet. The source term model reproduces velocity profiles very similar to the steady mass flow boundary condition, which is used to model steady micro jets. Unlike the steady mass flow boundary condition, the source term does not need a grid for the jet to capture the angle of attack and incidence. This feature will allow an investigator to quickly investigate varying angles of attacks and incidence of a steady micro jet without having to generate a grid each time.

The source term model is a viable tool for investigating steady micro jet installations on a surface. As large cases are investigated, the use of the model will significantly reduce the turnaround time from conception to solution by reducing grid generation and computational time.

\section{Acknowledgements}

The National Aeronautics and Space Administration Ultra Efficient Engine Technology Project sponsored this work. The author would like to acknowledge Pieter Buning for his help in implementing the source term model into OVERFLOW. Also Susan Gorton and Brian Allan are acknowledged for their help and guidance in this effort. The Configuration Aerodynamics Branch of the National Aeronautics and Space Administration Langley Research Center is acknowledged for their support on this project.

\section{References}

1. Hamstra, J. W., Miller, D. N., Truax, P. P., Anderson, B. H., and Wendt, B. J., Active Inlet Flow Control Technology Demonstration. ICAS-2000-6.11.2, 2000.

2. Gorton, S. A., Owens, L. R., Jenkins, L. N., Allan, B. G., Schuster, E. P, Active Flow Control On a Boundary-Layer-Ingesting Inlet. AIA A 2004-1203, January 2004.

3. Allan, B.G., Yao, C.S., and Lin, J.C, Numerical Simulations of Vortex Generator Vanes and Jets on a Flat Plate, AIAA Paper 2002-3160, June 2002.

4. Bender, E. E., Anderson, B. H., and Yagle, P. J., Vortex Generator Modeling for Navier-Stokes Codes. FEDSM99-6919, 1999.

5. Waithe, K. A., Source Term Model for Vortex Generator Vanes in a Navier-Stokes Computer Code, AIAA 2004-1236, January2004.

6. Buning, P.G., Jespersen, D. C., Pulliam, T. H., Klopfer, W.M., Chan, W. M., Slotnick, J. P., Krist, S. E., and Renze, K. J., OVERFLOW User's Manual Version I.8s, NASA Langley Research Center, Hampton, VA, November 2000.

7. Pulliam, T. H. and Chaussee, D. S., A Diagonal Form of an Implicit Approximate-Factorization Algorithm, Journal of Computational Physics, Vol. 39, No.2, pp. 347-363, 1981.

8. Steger, J. L., Dougherty, F. C., and Benek, J. A., A Chimera Grid Scheme, in Advances in Grid Generation, K. N. Ghia and U. Ghia, eds, vol. 5 of FED ASME, New York, NY, 1983.

9. Spalart, P. and Allmaras, S., A One-Equation Turbulence Model for Aerodynamic flows, La Recherche Aerospatiale, No.1, pp. 5-21, 1994. 